Submitted to ApJ LetTers

Preprint typeset using LTEX style emulateapj v. 5/2/11

\title{
DYNAMICAL VERSUS STELLAR MASSES IN COMPACT EARLY-TYPE GALAXIES: FURTHER EVIDENCE FOR SYSTEMATIC VARIATION IN THE STELLAR INITIAL MASS FUNCTION
}

\author{
Charlie Conroy $^{1,2}$, Aaron A. Dutton ${ }^{3}$, Genevieve J. Graves ${ }^{4}$, J. Trevor Mendel ${ }^{5}$, And Pieter G. VAn Dokkum ${ }^{6}$ \\ Submitted to ApJ Letters
}

\begin{abstract}
Several independent lines of evidence suggest that the stellar initial mass function (IMF) in early-type galaxies becomes increasingly 'bottom-heavy' with increasing galaxy mass and/or velocity dispersion, $\sigma$. Here we consider evidence for IMF variation in a sample of relatively compact early-type galaxies drawn from the Sloan Digital Sky Survey (SDSS). These galaxies are of sufficiently high stellar density that a dark halo likely makes a minor contribution to the total dynamical mass, $M_{\mathrm{dyn}}$, within one effective radius. We fit our detailed stellar population synthesis models to the stacked absorption line spectra of these galaxies in bins of $\sigma$ and find evidence from IMF-sensitive spectral features for a bottom-heavy IMF at high $\sigma$. We also apply simple 'mass-follows-light' dynamical models to the same data and find that $M_{\mathrm{dyn}}$ is significantly higher than what would be expected if these galaxies were stellar dominated and had a universal Milky Way IMF. Adopting $M_{\text {dyn }} \approx M_{*}$ therefore implies that the IMF is 'heavier' at high $\sigma$. Most importantly, the quantitative amount of inferred IMF variation is very similar between the two techniques, agreeing to within $\lesssim 0.1 \mathrm{dex}$ in mass. The agreement between two independent techniques, when applied to the same data, provides compelling evidence for systematic variation in the IMF as a function of early-type galaxy velocity dispersion. Any alternative explanations must reproduce both the results from dynamical and stellar population-based techniques.

Subject headings: galaxies: stellar content — galaxies: elliptical and lenticular, $\mathrm{cD}$ - galaxies: formation galaxies: fundamental parameters
\end{abstract}

\section{INTRODUCTION}

The stellar initial mass function (IMF) plays a fundamental role in studies of distant galaxies. Stars with masses near the main sequence turnoff dominate the luminosity of galaxies, and so an IMF is normally employed to extrapolate the properties of the turnoff stars (e.g., their number, age, and metallicity) to the more numerous, but fainter low mass stars. With an assumed IMF one can then estimate a variety of galactic properties, such as the total star formation rate and stellar mass. It is standard practice to assume that the IMF is universal (temporally and spatially invariant) and equal to either the Salpeter (1955) or Milky Way (MW) disk IMF (Kroupa 2001; Chabrier 2003) This assumption is supported by the lack of strong evidence for IMF variation within the MW (Bastian et al. 2010).

This lack of variation has been difficult to reconcile with theoretical expectations, as nearly every model for the IMF predicts at least some variation with physical properties, such as the temperature and pressure of molecular clouds (e.g., Larson 1998, 2005; Silk 1995; Adams \& Fatuzzo 1996; Padoan et al. 1997; Padoan \& Nordlund 2002; Hennebelle \& Chabrier 2008; Krumholz 2011; Hopkins 2012, 2013; Narayanan \& Davé 2012). However, despite the broad consensus that IMF vari-

\footnotetext{
${ }^{1}$ Department of Astronomy \& Astrophysics, University of California, Santa Cruz, CA, USA

2 Alfred P. Sloan Fellow

${ }^{3}$ Max Planck Institute for Astronomy, Königstuhl 17, 69117, Heidelberg, Germany

${ }^{4}$ Department of Astrophysical Sciences, Princeton University, Princeton, NJ, USA

${ }^{5}$ Max-Planck-Institute for Extraterrestrial Physics, Giessenbachstrasse, D-85748 Garching, Germany

${ }^{6}$ Department of Astrophysical Sciences, Yale University, New Haven, CT, USA

${ }^{7}$ For practical purposes, in this Letter we equate 'the MW IMF' with the Kroupa (2001) form.
}

ation should exist, there is little agreement on the main variables expected to control the variation, or even on the qualitative sense of the expected variation.

In the past several years two independent modeling techniques - stellar population synthesis (SPS) and dynamical - have pointed toward possible variation in the form of the IMF within the early-type/quiescent galaxy population. In particular, these techniques suggest that the IMF becomes increasingly 'bottom-heavy' (i.e., an IMF with a greater proportion of low-mass, $M \lesssim 1 M_{\odot}$, stars compared to the MW IMF) with increasing galaxy mass, velocity dispersion, and/or elemental abundance pattern (e.g., Cenarro et al. 2003; van Dokkum \& Conrov 2010; Treu et al. 2010; Auger et al. 2010; Graves \& Faber 2010; Thomas et al. 2011; Sonnenfeld et al. 2012; Cappellari et al. 2012; Dutton et al. 2012, 2013a b; Conroy \& van Dokkum 2012b; Ferreras et al. 2013; Smith et al.|2012; Spiniello et al. 2012, 2013; La Barbera et al. 2013; Tortora et al. 2013). At the same time, several studies have found evidence for 'bottom-light' IMFs in low mass systems (Strader et al.|2011; Zaritsky et al. 2013; Geha et al. 2013), and perhaps even in the Small Magellanic Cloud (Kalirai et al. 2013). In the most extreme cases the quoted variation in the IMF amongst massive early-type galaxies would imply a revision in the stellar masses upward by a factor of $3-4$.

Despite the broad qualitative agreement between two independent techniques, there is still some room for skepticism. Both dynamical and SPS techniques are subject to a variety of systematic uncertainties that are difficult to quantify. Only very recently have several groups begun to make direct comparisons between dynamical and SPS constraints on IMF variation (La Barbera et al. 2013; Tortora et al. 2013). The goal of this Letter is to directly compare dynamical and SPS-based mass-to-light ratios for a sample of compact early-type galaxies. We expect these galaxies to be stellar-dominated, at least 
Normal ETGs:
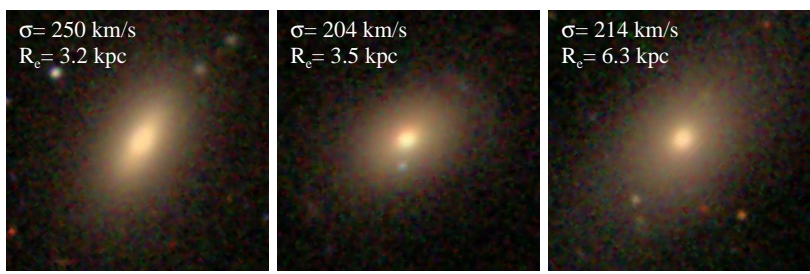

Compact ETGs:
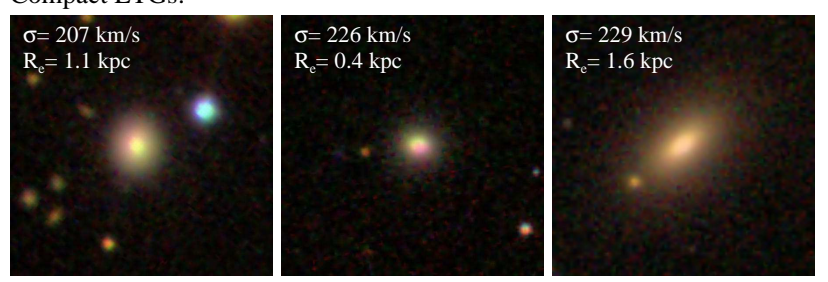

Figure 1. Representative gri composite images of early-type galaxies in a narrow $\sigma$ bin. Each image is $60^{\prime \prime} \times 60^{\prime \prime}$. These galaxies were chosen to reside in a narrow redshift interval, $0.03<z<0.04$, so that the relative apparent sizes are equal to relative physical sizes. The upper and lower panels compare normal and compact early-type galaxies (ETGs).

within an effective radius. Consideration of compact galaxies is therefore expected to reduce an important source of systematic uncertainty in the dynamical models (i.e., the inclusion of a dark halo).

The rest of this Letter is organized as follows. In Section 2 we describe the data and sample selection; in Section 3 we provide a brief overview of the dynamical and SPS techniques. The results are presented in Section 4 . Where necessary a flat $\Lambda C D M$ cosmology is assumed with the following parameters: $\left(\Omega_{\mathrm{m}}, \Omega_{\Lambda}, H_{0}\right)=(0.3,0.7,70)$.

\section{DATA \& SAMPLE SELECTION}

The sample of compact early-type galaxies analyzed in this Letter is drawn from the SDSS (York et al. 2000) Data Release 7 (Abazaiian et al. 2009). Stellar velocity dispersions, $\sigma$, are taken to be a weighted mean of the "SDSS" and "Princeton" spectroscopic pipelines. Total circularized half-light radii, $R_{\mathrm{e}}$, are measured via bulge plus disk decompositions provided in Simard et al. (2011). As a check on these half-light radii, we also consider half-light sizes provided by the SDSS photometric pipeline (specifically, the sizes based on a de Vaucouleurs light profile). All sizes are corrected for seeing. Photometric stellar mass-to-light ratios, $M_{*, \mathrm{MW}} / L$, are available for this sample from two sources: a catalog produced by one of us (Mendel et al. in prep), and a catalog provided by the MPA/JHU group (available at http://www.mpa-garching.mpg.de/SDSS/DR7//. The Mendel et al. stellar masses were derived by modeling the SDSS ugriz total magnitudes (based on the Simard et al. light profile fits) employing standard population synthesis techniques. In both catalogs, stellar mass-to-light ratios were also computed from the fiber-based photometry in order to compare directly with spectroscopically-based values. In the photometric analyses a Chabrier (2003) IMF was adopted. We have converted these masses to a Kroupa (2001) IMF by increasing them by $10 \%$, the approximate conversion between the two IMFs for old stellar populations. The quantity $M_{*, \mathrm{MW}}$ will throughout refer to stellar masses assuming a MW (Kroupa 2001) IMF.

We focus on compact galaxies in this work because there are several reasons to believe that they should be stellardominated at least within an effective radius. First, their den-
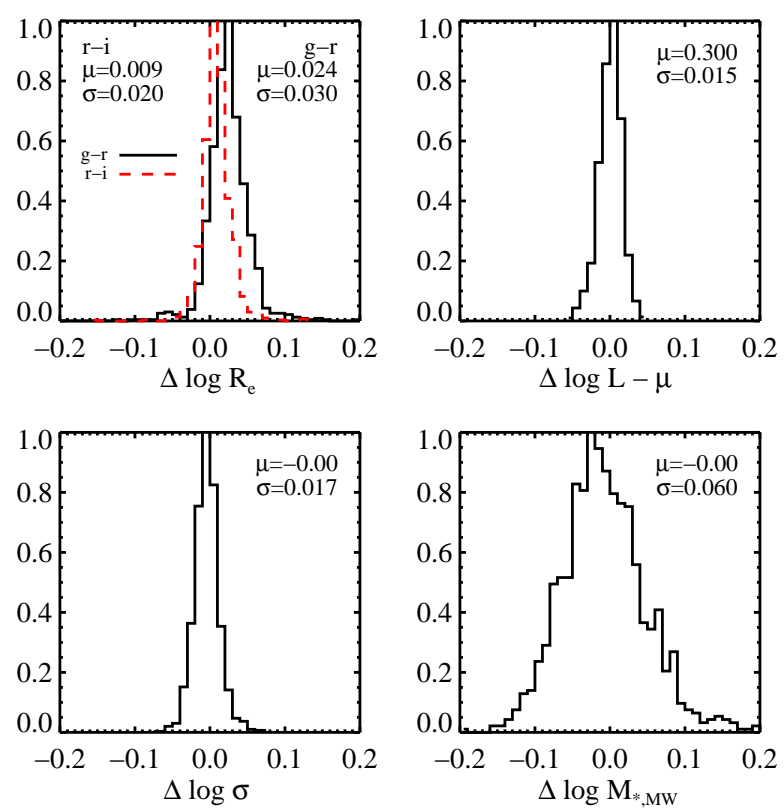

Figure 2. Stability of the physical properties of the sample. In each panel, the mean and standard deviation of each distribution is shown in the legend. Top Left: Difference in $R_{\mathrm{e}}$ between the $g, r$, and $i$ bands, as determined by the SDSS photometric pipeline assuming a de Vaucouleurs light profile. Top Right: Difference in total luminosity measured in the $g$ and $r$ bands. Bottom Left: Difference in velocity dispersion measured from the same spectra but with two different reduction pipelines (the "SDSS" and "Princeton" versions; see also Hyde \& Bernardi 2009). Bottom Right: Difference in stellar masses derived from photometry and assuming a Kroupa (2001) IMF, for two different fitting codes (Mendel et al. and MPA/JHU).

sities are so high that one would require substantial modification to a standard NFW (Navarro et al. 1997) dark matter density profile in order to achieve central dark matter densities high enough to rival the stellar densities. If these compact galaxies resided within NFW dark matter halos of mass $M_{h}=10^{12.5} M_{\odot}$ with a concentration of $c=6$ (expected from the $c(M)$ relation; Macciò et al. 2008), the dark matter fraction, $f_{\mathrm{DM}}$, within $R_{e}$ would be $5 \%$. Considering concentrations that lie $2 \sigma$ above the concentration-mass relation $(c=10)$ yields $f_{\mathrm{DM}}=10 \%$. Increasing the halo mass to $10^{13} M_{\odot}$ changes these fractions to $7 \%$ and $13 \%$. These numbers do not include the possibility of adiabatic contraction. We will show in Section 4 that a dark matter fraction of $65 \%$ would be required to explain the totality of the trend reported here. This is incompatible with kinematic models for these galaxies. They follow the virial fundamental plane, and their observed scaling relations and two-dimensional kinematics can only be fit with mass-follows light models, with an upper limit on the central DM fractions of $<20 \%$ (see Dutton et al. 2012, 2013a; Cappellari et al. 2013, for details). When modeling $M_{\text {dyn }}$ we make the simplifying assumption that these galaxies are stellar dominated and hence that "mass follows light".

Our sample of compact early-type galaxies was selected according to the following cuts. A redshift range of $0.025<z<$ 0.06 was imposed so that the reddest part of the CaT spectral feature is always in the observed window. Galaxies were selected to have a spectroscopic pipeline flag eCLASS $<0$, indicating an early-type spectrum, a red $g-r$ color $(g-r>$ $\left.0.59+0.052 \log \left[M_{*, \mathrm{MW}} / M_{\odot}-10\right]\right)$, and a minor-to-major axis ratio greater than 0.5 . These cuts result in a sample of 19,000 galaxies. We then computed stellar surface mass densities 
Table 1

Properties of Compact Early-Type Galaxies

\begin{tabular}{ccccccc}
\hline \hline $\log \sigma$ & $\mathrm{N}$ & $R_{\mathrm{fib}} / R_{\mathrm{e}}$ & $R_{\mathrm{e}}$ & $M_{*, \mathrm{MW}}$ & $M_{\mathrm{dyn}} / M_{*, \mathrm{MW}}$ & $M_{*} / M_{*, \mathrm{MW}}$ \\
\hline 1.96 & 34 & 1.9 & 0.6 & 10.0 & $0.98 \pm 0.05$ & $1.28 \pm 0.18$ \\
2.06 & 172 & 1.7 & 0.8 & 10.2 & $1.24 \pm 0.02$ & $1.35 \pm 0.09$ \\
2.15 & 352 & 1.5 & 0.9 & 10.4 & $1.55 \pm 0.02$ & $1.47 \pm 0.07$ \\
2.25 & 345 & 1.2 & 1.2 & 10.6 & $1.94 \pm 0.03$ & $1.69 \pm 0.06$ \\
2.34 & 169 & 1.0 & 1.4 & 10.7 & $2.31 \pm 0.05$ & $1.85 \pm 0.08$ \\
2.43 & 32 & 0.7 & 1.9 & 11.0 & $2.90 \pm 0.15$ & $2.27 \pm 0.16$
\end{tabular}

Note. - All quantities are averages within the bin except for $N$, the number of galaxies in each bin. Velocity dispersion, $\sigma$, is in units of $\log$ $\mathrm{km} \mathrm{s}^{-1}, R_{\mathrm{fib}} / R_{\mathrm{e}}$ is the average size of the fiber in units of the half-light radius, $r$-band half-light radius, $R_{\mathrm{e}}$, is in $\mathrm{kpc}$, and $M_{*, \mathrm{MW}}$ is quoted in units of $\log M_{\odot}$. Errors are $1 \sigma$ statistical uncertainties.

within $R_{e}$ according to: $\Sigma_{*}=M_{*, \mathrm{MW}}^{\text {tot }} /\left(2 \pi R_{\mathrm{e}}^{2}\right)$, where $R_{\mathrm{e}}$ is measured in the $r$-band and $M_{*, M W}^{\text {tot }}$ is the photometricallyderived total stellar mass assuming a MW IMF. Galaxies were selected to be compact via the cut $\Sigma_{*}>2500 M_{\odot} \mathrm{pc}^{-2}$. These cuts yield 1100 compact early-type galaxies $(\approx 6 \%$ of the early-type galaxy population) that define the sample used in the present analysis. In the following analysis these galaxies are grouped into six equally-spaced bins in $\log \sigma$ from $1.9<\log \sigma / \mathrm{km} \mathrm{s}^{-1}<2.5$. Several derived parameters in these $\sigma$ bins are shown in Table 1. Figure 1 shows gri composite images for both normal and compact early-type galaxies in a narrow bin in redshift and $\sigma$. On average, the compact galaxies are $2.5 \times$ smaller than the overall early-type population, implying that they are $\approx 15 \times$ denser (in terms of $M_{\odot} \mathrm{pc}^{-3}$ ).

These galaxies are remarkably dense and small; from the lowest to the highest $\sigma$ bins, the mean sizes range from 0.6 $\mathrm{kpc}$ to $2.3 \mathrm{kpc}$, corresponding approximately to $0.8^{\prime \prime}-2.3^{\prime \prime}$ at the median redshift of the sample (see Table 1). For reference, the SDSS pixel scale is $0.4^{\prime \prime}$ and the seeing is $1^{\prime \prime}-1.5^{\prime \prime}$. Only the largest galaxies in our sample are therefore (just) resolved within the half-light radius. Note however that half-light sizes can be reliably estimated even for such small galaxies because their total sizes subtend a considerably larger angle on the sky (see Figure 1).

The SDSS obtains spectra with fibers that have $3^{\prime \prime}$ diameters. Despite the fact that our sample is of relatively low redshift, their high surface densities imply that the SDSS fibers cover a significant fraction of the galaxy. From the lowest to the highest $\sigma$ bins the fiber covers on average the inner $1.9 R_{\mathrm{e}}$ to $0.7 R_{\mathrm{e}}$ (see Table 1). When fitting the data to SPS models we utilize spectra that are stacked in bins of $\sigma$. Each spectrum is continuum-normalized and broadened to a common dispersion of $\sigma=350 \mathrm{~km} \mathrm{~s}^{-1}$, and the stack is created by giving equal weight to each spectrum, after masking strong sky lines. The resulting stacked spectra have signal-to-noise ratios ranging from $\sim 100-700 \AA^{-1}$.

In Figure 2 we show the robustness of the parameters used to select the compact early-type galaxy sample. The key point is that sizes, luminosities, velocity dispersions, and stellar masses (with an assumed MW IMF) are all measured consistently between different images, in different bands, and/or using different photometric pipelines. Therefore, even though we have selected outliers (i.e., extremely com- pact galaxies), their properties are robust and not due to measurement errors. Note that the derivations of $M_{\mathrm{dyn}} / L$ shown below use the full galaxy light profiles, and thus do not depend explicitly on any of these measured parameters

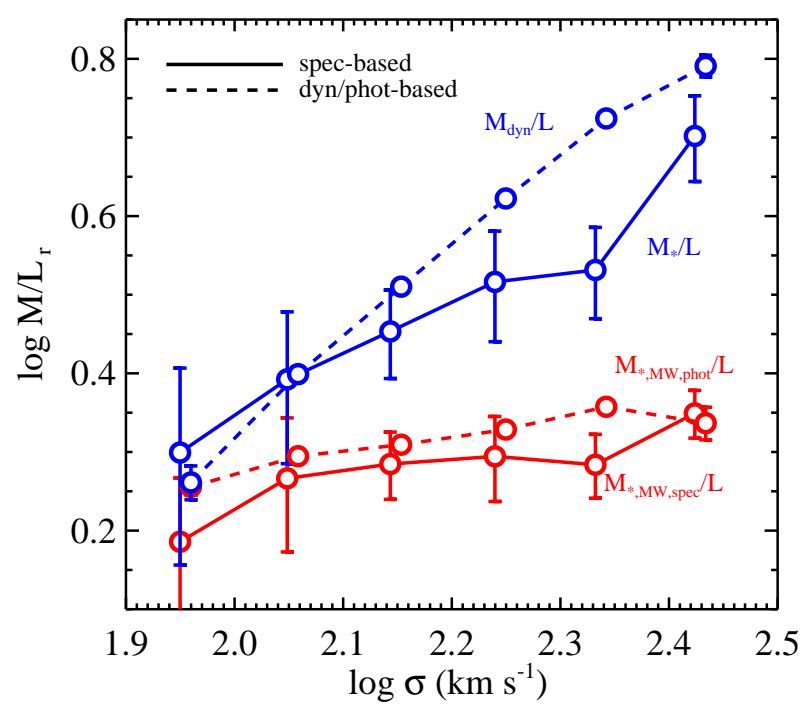

Figure 3. Mass-to-light ratio in the $r$-band, $M / L_{r}$, as a function of galaxy velocity dispersion, $\sigma$. Values obtained from fitting absorption line spectra (solid lines) are compared to values estimated from photometric SPS and dynamical modeling (dashed lines). Red lines are results for a fixed MW IMF, while blue lines make no assumption about the IMF. In particular, $M_{*} / L$ is the result of allowing the IMF to be constrained directly by the absorption line data. The difference between $M_{*} / L$ and $M_{*, \mathrm{MW} \text {,spec }} / L$ is due solely to differences in the IMF, while the difference between $M_{\mathrm{dyn}} / L$ and $M_{*, \mathrm{MW} \text {,phot }} / L$ can in principle be due to either the IMF or dark matter. Errors on the dashed lines are very small because they are errors on the mean, whereas the errors on the solid lines reflect the uncertainty from fitting stacked spectra.

\section{METHODS}

In this section we describe the techniques for computing $M_{\mathrm{dyn}} / L$ and $M_{*} / L$ for our sample of compact early-type galaxies.

Stellar mass-to-light ratios are computed by fitting an SPS model to the stacked absorption line spectra of the compact early-type galaxies. The SPS model used herein was developed in Conroy \& van Dokkum (2012a.b). In its present form the model contains 27 free parameters, including the redshift and velocity dispersion, a two-part power-law IMF, two population ages, four nuisance parameters, and the abundances of 17 elements. These parameters are fit to the data via a Markov Chain Monte Carlo fitting technique. For the purposes of this Letter the most important feature is that the IMF is constrained directly by the data, in particular by a variety of spectral features that lie mainly in the red spectral range (e.g., numerous $\mathrm{TiO}$ bands at $>6000 \AA$, $\mathrm{Na}$ I at $8200 \AA$ and Ca II at $8500-8670 \AA$ ). The data and models are split into four wavelength intervals and within each interval the spectra are normalized by a high-order polynomial (see Conroy \& van Dokkum 2012b; Conroy et al. 2013, for details). The main result of this fitting procedure is an estimate of the true stellar mass-to-light ratio, $M_{*} / L$; 'true' because the IMF is included as a parameter determined by the data. For each set of model parameters we also keep track of the stellar mass-to-light ratio that would be expected assuming a MW (Kroupa 2001) IMF, $M_{*, \mathrm{MW}} / L$. Notice that the ratio between the measured stellar mass and the stellar mass assuming a MW IMF, $M_{*} / M_{*, \mathrm{MW}}$, is sensitive only to the IMF. The resulting best-fit parameters are very well-constrained, with essentially no degeneracies between the IMF and other parameters. Quoted errors are $1 \sigma$ limits marginalized over the 


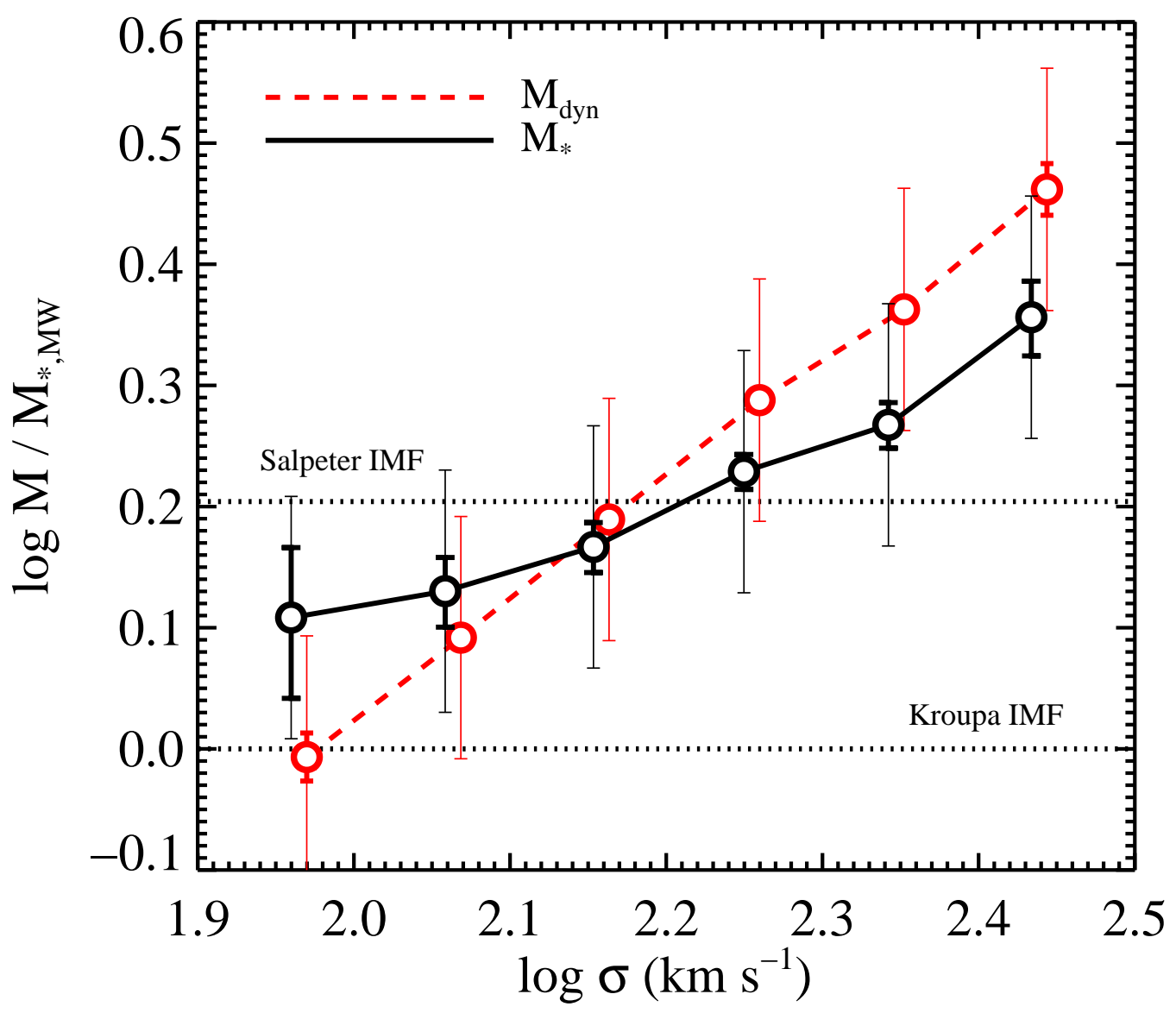

Figure 4. Variation in the normalized mass with galaxy velocity dispersion, $\sigma$, where 'normalized mass' is defined as the measured mass in units of the stellar mass derived assuming a MW (Kroupa 2001) IMF. Dynamical masses (dashed line) are compared to stellar population-based masses (solid line). For the latter, the $y$-axis is sensitive only to the IMF, while for the former the $y$-axis is in principle sensitive to both IMF variation and dark matter. Dotted lines show the expected values if the galaxies had Kroupa (2001) or Salpeter (1955) IMFs. Thick error bars represent formal statistical errors, while the thin error bars represent 0.1 dex systematic errors. The two curves are shifted slightly along the $x$-axis for clarity.

full posterior distribution.

For each galaxy we calculate $M_{\text {dyn }}$ and $M_{\text {dyn }} / L$ from the following procedure (see Dutton et al. 2012, for details). We start with a galaxy light profile parameterized by circularized and de-projected Sérsic $n=4$ and $n=1$ components. Then, assuming that mass follows light, we calculate the radial velocity dispersion profile by solving the spherical Jeans equations assuming isotropic orbits $(\beta=0$; e.g., Binney \& Mamon 1982) and a purely pressure-supported system. As discussed in Dutton et al. (2012), due to the large fraction of the galaxy light covered by the fiber, the dynamical masses are only weakly dependent on stellar anisotropy (see also Wolf et al. 2010). Adopting $\beta=0.5$ changes $M_{\text {dyn }}$ by $<5 \%$. We then compute the projected velocity dispersion (convolved with $1.4^{\prime \prime}$ FWHM seeing, typical for the SDSS) within the $3^{\prime \prime}$ diameter aperture used by SDSS. Finally, we compute $M_{\text {dyn }}$ by scaling the model aperture velocity dispersion to match the observed aperture velocity dispersion. Notice that the dynamical masses are completely independent of the SPS stellar masses, and since mass follows light, $M_{\text {dyn }} / L$ is constant with radius. We then compute the mean $M_{\text {dyn }} / L$ within the SDSS fiber aperture in bins of $\sigma$. In addition to the formal statistical error, we also assign a systematic error of 0.1 dex to the dynamical masses. The motivation for this error comes in part from Figure 2, where sizes and dispersion can differ by $0.02-0.06$ dex depending on assumptions. In addition, uncertainties in the anisotropy profile, degree of rotational support, etc., when combined can be expected to affect $M_{\text {dyn }} / L$ at the 0.1 dex level.

There are four distinct 'mass-to-light ratios' in this Letter, which we summarize here for clarity. $M_{\text {dyn }} / L$ is based on a dynamical model; $M_{*} / L$ is reserved for the 'true' stellar massto-light ratio inferred from spectral fitting when the IMF is left as a free parameter; $M_{*, \mathrm{MW}} / L$ is, generically, the stellar massto-light ratio assuming a MW IMF. In the present work there are two estimates of this last quantity; the first from fitting ugriz photometry, and the second from fitting the absorption line spectra with the IMF fixed to the MW form. In practice these two techniques provide nearly identical estimates of $M_{*, \mathrm{Mw}} / L$, so we do not distinguish between these two except where necessary.

Finally, notice that all quoted mass-to-light ratios are determined within the same aperture, specifically within the $3^{\prime \prime}$ SDSS fiber. 'Total' mass-to-light ratios and masses are only used for selecting the sample. 


\section{RESULTS}

The best-fit mass-to-light ratios, $M / L$, for the three techniques - photometric, spectroscopic, and dynamical — are shown in Figure 3. The red lines compare photometrically and spectroscopically derived $M_{*, \mathrm{MW}} / L$ values, and it is encouraging that they agree well. The blue lines compare the results from dynamical models to the results from the spectroscopic model, where in the latter case the IMF is a free variable derived from the data. All $M / L$ ratios in this figure were derived within the same aperture (the spectroscopic fiber). As we argued in Section 2 the expected contribution from dark matter should be small in these compact galaxies, certainly less than the dark matter fractions of $\approx 65 \%$ that would be required to attribute entirely to dark matter the difference between $M_{\text {dyn }} / L$ and $M_{*, \mathrm{MW}} / L$ at high $\sigma$.

The main result of this Letter is shown in Figure 4. In this figure we compare the best-fit dynamical and SPS-based masses as a function of galaxy velocity dispersion, $\sigma$. The key feature of this diagram is that masses are plotted in units of the stellar mass assuming a MW IMF, $M_{*, \mathrm{MW}}$. The $y$-axis is thus insensitive to normal stellar population variation such as stellar age, metallicity, etc. Dotted lines show the expected ratios if the galaxies had Kroupa (2001) or Salpeter (1955) IMFs. In general $M_{\text {dyn }}$ will be a mix of stellar mass and dark halo mass, but as we argued in Section 2, the galaxies analyzed herein are sufficiently dense that $M_{\mathrm{dyn}}$ is likely dominated by the stars. We also emphasize that $M_{*} / L$ and $M_{\text {dyn }} / L$ are completely independent in the sense that the former is derived from absorption line spectra while the latter is derived from a combination of $R_{\mathrm{e}}, L, \sigma$, and the detailed light profile shape. Furthermore, while formally the axes are correlated for the $M_{\text {dyn }} / M_{*, \text { MW }}$ vs. $\sigma$ relation $\left(M_{\text {dyn }} \propto \sigma^{2}\right)$, we have simulated the effect of measurement errors and find that the nominal measurement errors (Figure 2) have a small effect on the result. If the true random errors were as large as $0.05 \mathrm{dex}$, then half of the difference between the $M_{\text {dyn }}$ and $M_{*}$ relations could be explained by the correlated axes in the former relation.

The key result is that $M_{\mathrm{dyn}} / M_{*, \mathrm{MW}}$ and $M_{*} / M_{*, \mathrm{MW}}$, and their variation with $\sigma$, agree very well, perhaps even better than expected given expected systematic errors that could plausibly exceed $0.1 \mathrm{dex}$. In addition, both masses point toward an IMF that becomes increasingly bottom-heavy with increasing $\sigma$. At the highest dispersions the inferred IMF becomes considerably steeper than even the Salpeter IMF.

We emphasize that not only were the dynamical, photometric and spectroscopic masses derived for the same set of galaxies, but they were also derived for data obtained within the exact same aperture, i.e., the (seeing-convolved) SDSS fiber. Figure 4 therefore presents the most direct comparison of spectral and dynamically-based IMF results to-date.

We caution that the quantitative trend shown here for our sample of 1100 compact early-type galaxies may not to be representative of the entire early-type galaxy population, and in fact there are emerging clues that galaxy compactness may be an important variable governing the IMF shape (Läsker et al. 2013; Smith \& Lucey 2013). In a forthcoming analysis we will explore the two dimensional manifold of IMF variation as a function of $\sigma$ and galaxy compactness (e.g., $\Sigma_{*}$ and/or $R_{\mathrm{e}}$ ).

The fact that the same IMF variation is seen both via dynamical and SPS-based techniques is reassuring and suggests that neither technique suffers from catastrophic systematic errors. However, we caution that consistency does not neces- sarily imply that both techniques are correct, as independent systematic errors in the mass measurements could work in the same direction. Addressing possible sources of systematic uncertainty is the subject of ongoing work.

CC acknowledges support from the Alfred P. Sloan Foundation. This paper is based on data from the Sloan Digital Sky Survey.

\section{REFERENCES}

Abazajian, K. N., Adelman-McCarthy, J. K., Agüeros, M. A., Allam, S. S., Allende Prieto, C., An, D., Anderson, K. S. J., Anderson, S. F., Annis, J., Bahcall, N. A., \& et al. 2009, ApJS, 182, 543

Adams, F. C. \& Fatuzzo, M. 1996, ApJ, 464, 256

Auger, M. W., Treu, T., Gavazzi, R., Bolton, A. S., Koopmans, L. V. E., \& Marshall, P. J. 2010, ApJ, 721, L163

Bastian, N., Covey, K. R., \& Meyer, M. R. 2010, ARA\&A, 48, 339

Binney, J. \& Mamon, G. A. 1982, MNRAS, 200, 361

Cappellari, M., Scott, N., Alatalo, K., et al. 2013, MNRAS, 432, 1709

Cappellari, M. et al. 2012, Nature, 484, 485

Cenarro, A. J., Gorgas, J., Vazdekis, A., Cardiel, N., \& Peletier, R. F. 2003 MNRAS, 339, L12

Chabrier, G. 2003, PASP, 115, 763

Conroy, C. \& van Dokkum, P. 2012a, ApJ, 747, 69

Conroy, C. \& van Dokkum, P. G. 2012b, ApJ, 760, 71

Conroy, C., van Dokkum, P. G., \& Graves, G. J. 2013, ApJ, 763, L25

Dutton, A. A., Macciò, A. V., Mendel, J. T., \& Simard, L. 2013a, MNRAS, 432, 2496

Dutton, A. A., Mendel, J. T., \& Simard, L. 2012, MNRAS, 422, L33

Dutton, A. A. et al. 2013b, MNRAS, 428, 3183

Ferreras, I., La Barbera, F., de la Rosa, I. G., Vazdekis, A., de Carvalho,

R. R., Falcón-Barroso, J., \& Ricciardelli, E. 2013, MNRAS, 429, L15

Geha, M., Brown, T. M., Tumlinson, J., et al. 2013, ApJ, 771, 29

Graves, G. J. \& Faber, S. M. 2010, ApJ, 717, 803

Hennebelle, P. \& Chabrier, G. 2008, ApJ, 684, 395

Hopkins, P. F. 2012, MNRAS, 423, 2037

-. 2013, MNRAS, 433, 170

Hyde, J. B. \& Bernardi, M. 2009, MNRAS, 394, 1978

Kalirai, J. S. et al. 2013, ApJ, 763, 110

Kroupa, P. 2001, MNRAS, 322, 231

Krumholz, M. R. 2011, ApJ, 743, 110

La Barbera, F., Ferreras, I., Vazdekis, A., de la Rosa, I. G., de Carvalho, R. R., Trevisan, M., Falcón-Barroso, J., \& Ricciardelli, E. 2013, MNRAS, 433, 3017

Larson, R. B. 1998, MNRAS, 301, 569

-. 2005, MNRAS, 359, 211

Läsker, R., van den Bosch, R. C. E., van de Ven, G., Ferreras, I., La Barbera, F., Vazdekis, A., \& Falcón-Barroso, J. 2013, MNRAS, 434, L31

Macciò, A. V., Dutton, A. A., \& van den Bosch, F. C. 2008, MNRAS, 391, 1940

Narayanan, D. \& Davé, R. 2012, MNRAS, 423, 3601

Navarro, J. F., Frenk, C. S., \& White, S. D. M. 1997, ApJ, 490, 493

Padoan, P. \& Nordlund, A. 2002, ApJ, 576, 870

Padoan, P., Nordlund, A., \& Jones, B. J. T. 1997, MNRAS, 288, 145

Salpeter, E. E. 1955, ApJ, 121, 161

Silk, J. 1995, ApJ, 438, L41

Simard, L., Mendel, J. T., Patton, D. R., Ellison, S. L., \& McConnachie,

A. W. 2011, ApJS, 196, 11

Smith, R. J. \& Lucey, J. R. 2013, MNRAS, 434, 1964

Smith, R. J., Lucey, J. R., \& Carter, D. 2012, MNRAS, 426, 2994

Sonnenfeld, A., Treu, T., Gavazzi, R., Marshall, P. J., Auger, M. W., Suyu,

S. H., Koopmans, L. V. E., \& Bolton, A. S. 2012, ApJ, 752, 163

Spiniello, C., Trager, S., Koopmans, L. V. E., \& Conroy, C. 2013,

arXiv: 1305.2873

Spiniello, C., Trager, S. C., Koopmans, L. V. E., \& Chen, Y. P. 2012, ApJ, 753, L32

Strader, J., Caldwell, N., \& Seth, A. C. 2011, AJ, 142, 8

Thomas, J., Saglia, R. P., Bender, R., Thomas, D., Gebhardt, K., Magorrian,

J., Corsini, E. M., Wegner, G., \& Seitz, S. 2011, MNRAS, 415, 545

Tortora, C., Romanowsky, A. J., \& Napolitano, N. R. 2013, ApJ, 765, 8

Treu, T., Auger, M. W., Koopmans, L. V. E., Gavazzi, R., Marshall, P. J., \&

Bolton, A. S. 2010, ApJ, 709, 1195

van Dokkum, P. G. \& Conroy, C. 2010, Nature, 468, 940 
Wolf, J., Martinez, G. D., Bullock, J. S., Kaplinghat, M., Geha, M., Muñoz,

R. R., Simon, J. D., \& Avedo, F. F. 2010, MNRAS, 406, 1220

York, D. G. et al. 2000, AJ, 120, 1579

Zaritsky, D., Colucci, J. E., Pessev, P. M., Bernstein, R. A., \& Chandar, R. 2013, ApJ, 770, 121 